PROCEEDINGS OF THE

AMERICAN MATHEMATICAL SOCIETY

Volume 131, Number 5, Pages 1433-1442

S 0002-9939(02)06781-3

Article electronically published on September 19, 2002

\title{
ENDPOINT ESTIMATES \\ FOR THE CIRCULAR MAXIMAL FUNCTION
}

\author{
SANGHYUK LEE
}

(Communicated by Andreas Seeger)

\begin{abstract}
We consider the problem of endpoint estimates for the circular maximal function defined by$$
M f(x)=\sup _{1<t<2}\left|\int_{S^{1}} f(x-t y) d \sigma(y)\right|
$$

where $d \sigma$ is the normalized surface area measure on $S^{1}$. Let $\Delta$ be the closed triangle with vertices $(0,0),(1 / 2,1 / 2),(2 / 5,1 / 5)$. We prove that for $(1 / p, 1 / q) \in$ $\Delta \backslash\{(1 / 2,1 / 2),(2 / 5,1 / 5)\}$, there is a constant $C$ such that $\|M f\|_{L^{q}\left(\mathbb{R}^{2}\right)} \leq$ $C\|f\|_{L^{p}\left(\mathbb{R}^{2}\right)}$. Furthermore, $\|M f\|_{L^{5, \infty}\left(\mathbb{R}^{2}\right)} \leq C\|f\|_{L^{5 / 2,1}\left(\mathbb{R}^{2}\right)}$.
\end{abstract}

\section{Introduction And STATEMENT Of RESUlts}

Bourgain [B1] showed that the circular maximal function defined by

$$
\sup _{t>0}\left|\int_{S^{1}} f(x-t y) d \sigma(y)\right|
$$

is bounded on $L^{p}\left(\mathbb{R}^{2}\right)$ if $p>2$. Mockenhaupt, Seeger and Sogge [MSS] later found a new proof of this result based on their local smoothing estimates. Their result actually implies that if one modifies the definition so that the supremum is taken over $1<t<2$, then the resulting maximal operator $M$ (see below) is bounded from $L^{p}\left(\mathbb{R}^{2}\right)$ to $L^{q}\left(\mathbb{R}^{2}\right)$ for some $q>p$. Here $M$ is defined by

$$
M f(x)=\sup _{1<t<2}\left|\int_{S^{1}} f(x-t y) d \sigma(y)\right| .
$$

Let $\Delta$ be the closed triangle with vertices $P=(2 / 5,1 / 5), Q=(1 / 2,1 / 2), R=(0,0)$. Schlag $\left[\mathbf{S}\right.$ ] showed $M$ is bounded from $L^{p}\left(\mathbb{R}^{2}\right) \rightarrow L^{q}\left(\mathbb{R}^{2}\right)$ if $(1 / p, 1 / q)$ lies in the interior of $\Delta$. His result was obtained using a combinatorial method. A different proof was later obtained by Schlag and Sogge [SS] which was based on some local smoothing estimates. It can easily be shown that $M$ cannot be bounded from $L^{p}\left(\mathbb{R}^{2}\right)$ to $L^{q}\left(\mathbb{R}^{2}\right)$ if $(1 / p, 1 / q) \in([0,1] \times[0,1] \backslash \Delta) \cup\{(1 / 2,1 / 2)\}$ (see [S], [SS]). Thus, when $(1 / p, 1 / q) \in(R, P] \cup[P, Q)$, the $L^{p}-L^{q}$ estimates for $M$ are still open. In this note these remaining endpoint estimates are considered. The following is

Received by the editors June 12, 2001 and, in revised form, December 7, 2001.

2000 Mathematics Subject Classification. Primary 42B25; Secondary 35L05.

Key words and phrases. Circular maximal function, endpoint estimates.

The author was partially supported by the BK21 Project (PI: Jong-Guk Bak). 
our main result which gives the complete type set for $M$ except for the $L^{5 / 2}-L^{5}$ estimate.

Theorem 1.1. Let $M$ be defined by (1.1). Then for $(1 / p, 1 / q) \in \Delta \backslash\{P, Q\}$, there is a constant $C$ such that

$$
\|M f\|_{L^{q}\left(\mathbb{R}^{2}\right)} \leq C\|f\|_{L^{p}\left(\mathbb{R}^{2}\right)} .
$$

Furthermore $\|M f\|_{L^{5, \infty}\left(\mathbb{R}^{2}\right)} \leq C\|f\|_{L^{5 / 2,1\left(\mathbb{R}^{2}\right)}}$.

Theorem 1.1 will be proven using some sharp Carleson-Sjölin type estimate for the 2-dimensional wave equation. Let us define

$$
U_{t} f(x)=\int_{\mathbb{R}^{2}} e^{i\langle x, \xi\rangle+i t|\xi|} \widehat{f}(\xi) d \xi .
$$

In Section 2, we will show the following $L^{p}-L^{q}$ local smoothing estimates.

Proposition 1.2. If supp $\widehat{f} \subset\left\{\xi \in \mathbb{R}^{2}:|\xi| \sim N\right\}$, then for $1 / p+3 / q=1$, $14 / 3<q \leq \infty$,

$$
\left(\int_{\mathbb{R}^{2}} \int_{1}^{2}\left|U_{t} f(x)\right|^{q} d t d x\right)^{1 / q} \leq C N^{3 / 2-6 / q}\|f\|_{L^{p}\left(\mathbb{R}^{2}\right)} .
$$

This is a slight improvement of the results obtained by Schlag and Sogge [SS], and Tao and Vargas [TV2, section 4]. In particular, the $\epsilon$-loss on regularity is removed. For the proof of (1.2) we use the bilinear cone restriction estimate of Wolff [W] and Tao [T] together with a modification of an argument in [TV2, section 4]. Let $\Gamma=\left\{(\xi, \tau) \in \mathbb{R}^{2} \times \mathbb{R}:|\xi|=\tau, \quad 1 \leq \tau \leq 2\right\}$ and let $\Omega_{1}, \Omega_{2}$ be closed subsets of $S^{1}$ with $\operatorname{dist}\left(\Omega_{1}, \Omega_{2}\right)$ comparable to 1 . Now set $\Gamma_{i}=\left\{(\xi, \tau) \in \Gamma: \xi / \tau \in \Omega_{i}\right\}$ for $i=1,2$. The following is the bilinear cone restriction estimate in $\mathbb{R}^{3}$ due to Wolff W] (for $r>5 / 3$ ) and Tao [T] (for $r=5 / 3$ ):

If supp $f \subset \Gamma_{1}$ and supp $g \subset \Gamma_{2}$, then for $r \geq 5 / 3$

$$
\|\widehat{f d \mu} \widehat{g d \mu}\|_{r} \leq C\|f\|_{2}\|g\|_{2}
$$

where $d \mu$ is the surface measure of $\Gamma$.

Once Proposition 1.2 has been established, the proof of Theorem 1.1 is straightforward.

Proof of Theorem 1.1. It is well-known that

$$
\widehat{d \sigma}(\xi)=e^{i|\xi|} a_{+}(\xi)+e^{-i|\xi|} a_{-}(\xi)
$$

where $a_{ \pm}$are smooth functions satisfying $\left|\partial^{\alpha} a_{ \pm}(\xi)\right| \leq C_{\alpha}(1+|\xi|)^{-1 / 2-|\alpha|}$. Therefore, it is sufficient to show that the maximal operator $\mathcal{M}$ defined by

$$
\mathcal{M} f(x)=\sup _{1<t<2}\left|\int e^{i\langle x, \xi\rangle+i t|\xi|} a_{+}(t \xi) \widehat{f}(\xi) d \xi\right|
$$

satisfies the estimates in Theorem 1.1 in place of $M$. Let $\beta \in C_{0}^{\infty}(1 / 2,2)$ satisfying $\sum \beta\left(\cdot / 2^{j}\right)=1$ and let $\beta_{j}(\xi)=\beta\left(|\xi| / 2^{j}\right)$. Let $f_{j}, f_{0}$ be defined by $\widehat{f}_{j}=\beta_{j} \widehat{f}$, $f_{0}=\sum_{j \leq 0} f_{j}$, respectively. Set $\mathcal{M}_{j} f=\mathcal{M} f_{j}$. Trivially, we have

$$
\mathcal{M} f(x) \leq \mathcal{M} f_{0}(x)+\sum_{j \geq 1} \mathcal{M}_{j} f(x) .
$$

It is easy to see that $\left\|\mathcal{M} f_{0}\right\|_{q} \leq C\|f\|_{p}$ for $1 \leq p \leq q$, so we only need to consider $\sum_{j \geq 1} \mathcal{M}_{j} f$. Now we need the following well-known lemma. 
Lemma 1.3. Let $I$ be an interval and let $F$ be a smooth function defined on $\mathbb{R}^{n} \times I$. Then, for $1<p \leq \infty$,

$$
\left\|\sup _{t \in I}|F(x, t)|\right\|_{L^{p}\left(\mathbb{R}^{n}\right)} \leq C\left(\|F\|_{L^{p}\left(\mathbb{R}^{n} \times I\right)}+\|F\|_{L^{p}\left(\mathbb{R}^{n} \times I\right)}^{(p-1) / p}\left\|\partial_{t} F\right\|_{L^{p}\left(\mathbb{R}^{n} \times I\right)}^{1 / p}\right) .
$$

By Lemma 1.3 and Plancherel's theorem it is easy to see that for $j \geq 1$,

$$
\left\|\mathcal{M}_{j} f\right\|_{2} \leq C\|f\|_{2} \text {. }
$$

Let $I$ be the interval $[1,2]$. Using Lemma 1.3 and Hölder's inequality, we have

$\left\|\mathcal{M}_{j} f\right\|_{q} \leq C 2^{-j / 2}\left\|U_{t} f_{j}^{1}\right\|_{L^{q}\left(\mathbb{R}^{2} \times I\right)}+C 2^{-j / 2+j / q}\left(\left\|U_{t} f_{j}^{2}\right\|_{L^{q}\left(\mathbb{R}^{2} \times I\right)}+\left\|U_{t} f_{j}^{3}\right\|_{L^{q}\left(\mathbb{R}^{2} \times I\right)}\right)$

where the supports of the Fourier transforms of $f_{j}^{1}, f_{j}^{2}$ and $f_{j}^{3}$ are contained in the set $\left\{\xi \in \mathbb{R}^{2}:|\xi| \sim 2^{j}\right\}$, and $\left\|f_{j}^{1}\right\|_{p},\left\|f_{j}^{2}\right\|_{p}$ and $\left\|f_{j}^{3}\right\|_{p}$ are bounded by $C\|f\|_{p}$. Applying Proposition 1.2 to the last inequality, it is easy to see that for $1 / p+3 / q=1$ and $q>14 / 3$,

$$
\left\|\mathcal{M}_{j} f\right\|_{q} \leq C 2^{j(1-5 / q)}\|f\|_{p}
$$

A complex interpolation between this and (1.4) shows that if $(1 / p, 1 / q)$ is contained in the closed triangle with vertices $(1,0),(5 / 14,3 / 14),(1 / 2,1 / 2)$ but is not on the closed line segment $[(1 / 2,1 / 2),(5 / 14,3 / 14)]$, then

$$
\left\|\mathcal{M}_{j} f\right\|_{q} \leq C 2^{j(3 / p-1 / q-1) / 2}\|f\|_{p} .
$$

Using (1.5) and Lemma 2.6 (in Section 2) with $n=1$, we have for $(1 / p, 1 / q) \in$ $[P, Q)$,

$$
\|\mathcal{M} f\|_{q, \infty} \leq C\|f\|_{p, 1} .
$$

Since $\mathcal{M}$ is a local operator, an interpolation (real interpolation) between these estimates and the trivial $L^{\infty}-L^{\infty}$ estimate completes the proof of Theorem 1.1.

We point out that similar endpoint estimates hold for the spherical maximal function in $\mathbb{R}^{n}, n \geq 3$. Set

$$
M^{n} f(x)=\sup _{1<t<2}\left|\int_{S^{n-1}} f(x-t y) d \sigma^{n}(y)\right|
$$

where $d \sigma^{n}$ is the normalized surface area measure on $S^{n-1}$. Set $P_{1}=(0,0), P_{2}=$ $((n-1) / n,(n-1) / n), P_{3}=((n-1) / n, 1 / n), P_{4}=\left(\left(n^{2}-n\right) /\left(n^{2}+1\right),(n-1) /\left(n^{2}+1\right)\right)$. Let $\mathcal{Q}$ be the closed quadrangle with vertices $P_{1}, P_{2}, P_{3}, P_{4}$. It was shown in [SS] that $\left\|M^{n} f\right\|_{q} \leq C\|f\|_{p}$ if $(1 / p, 1 / q)$ is contained in the interior of $\mathcal{Q}$ and that these maximal inequalities can never hold if $(1 / p, 1 / q)$ is outside of $\mathcal{Q}$. Using an argument similar to the one used for the circular maximal function, we can show the following.

Theorem 1.4. Suppose $M^{n}$ is defined as in the above for $n \geq 3$. Then there is a constant $C$ such that

$$
\left\|M^{n} f\right\|_{L^{q}\left(\mathbb{R}^{n}\right)} \leq C\|f\|_{L^{p}\left(\mathbb{R}^{n}\right)}
$$

if $(1 / p, 1 / q)$ is contained in $\mathcal{Q} \backslash\left\{P_{2}, P_{3}, P_{4}\right\}$. Furthermore,

$$
\left\|M^{n} f\right\|_{L^{q, \infty}\left(\mathbb{R}^{n}\right)} \leq C\|f\|_{L^{p, 1}\left(\mathbb{R}^{n}\right)}
$$

if $(1 / p, 1 / q)=P_{2}, P_{3}, P_{4}$. 
Proof of Theorem 1.4. Now recall $\widehat{d \sigma^{n}}(\xi)=e^{i|\xi|} a_{+}(\xi)+e^{-i|\xi|} a_{-}(\xi)$ with $\left|\partial_{\xi}^{\alpha} a_{ \pm}(\xi)\right|$ $\leq C_{\alpha}(1+|\xi|)^{-(n-1) / 2-|\alpha|}$. Define

$$
\mathcal{M} f(x)=\sup _{1<t<2}\left|\int_{\mathbb{R}^{n}} e^{i\langle x, \xi\rangle+i t|\xi|} \frac{\widehat{f}(\xi)}{(1+|\xi|)^{(n-1) / 2}} d \xi\right| .
$$

It is sufficient to consider $\mathcal{M}$ instead of $M^{n}$. As before, by Littlewood-Paley decomposition, $f=f_{0}+\sum_{j \geq 1} f_{j}$. Set $\mathcal{M}_{j} f=\mathcal{M}\left(f_{j}\right)$. It can be easily seen that $\left\|\mathcal{M}_{0} f\right\|_{q} \leq C\|f\|_{p}$ for $1 \leq p \leq q$. Theorem 1.4 follows from Lemma 2.6 and the following estimates. There is a constant $C$ such that for $j \geq 1$,

$$
\begin{aligned}
\left\|\mathcal{M}_{j} f\right\|_{1} & \leq C 2^{j}\|f\|_{1}, \\
\left\|\mathcal{M}_{j} f\right\|_{\infty} & \leq C 2^{j}\|f\|_{1}, \\
\left\|\mathcal{M}_{j} f\right\|_{2} & \leq C 2^{-j \frac{n-2}{2}}\|f\|_{2}, \\
\left\|\mathcal{M}_{j} f\right\|_{2(n+1) /(n-1)} & \leq C 2^{-j \frac{n^{2}-2 n-1}{2 n+2}}\|f\|_{2} .
\end{aligned}
$$

Interpolations (by Lemma 2.6) between (1.7) and (1.9), (1.8) and (1.9), (1.8) and (1.10) give the restricted weak types $(n /(n-1), n /(n-1)),(n /(n-1), n)$, $\left(\left(n^{2}+1\right) /\left(n^{2}-n\right),\left(n^{2}+1\right) /(n-1)\right)$, respectively. Therefore, we only need to show (1.7), (1.8), (1.9), (1.10).

It is easy to see that (1.8) and (1.9) follow from the fact that if $\beta \in C_{0}^{\infty}\left(\mathbb{R}^{n} \backslash 0\right)$,

$$
\left|\int_{\mathbb{R}^{n}} e^{i\langle x, \xi\rangle+i t|\xi|} \frac{\beta\left(\xi / 2^{j}\right)}{(1+|\xi|)^{(n-1) / 2}} d \xi\right| \leq C \min \left(2^{j}, 2^{j(n+1) / 2}\left(1+2^{j}|| x|-t|\right)^{-N}\right)
$$

for every $N$. Using Lemma 1.3 and Plancherel's theorem, we have (1.9). To see (1.10), let $U_{t} f(x)=\int_{\mathbb{R}^{n}} e^{i\langle x, \xi\rangle+i t|\xi|} \widehat{f}(\xi) d \xi$ and use Lemma 1.3 to get

$$
\begin{aligned}
\left\|\mathcal{M}_{j} f\right\|_{L^{\frac{2 n+2}{n-1}\left(\mathbb{R}^{n}\right)}} \leq C 2^{-j \frac{n-1}{2}}\left\|U_{t} f_{j}^{1}\right\|_{L^{\frac{2 n+2}{n-1}}\left(\mathbb{R}^{n} \times I\right)} \\
+C 2^{-j \frac{n-1}{2}} 2^{j \frac{n-1}{2 n+2}}\left(\left\|U_{t} f_{j}^{2}\right\|_{L^{\frac{2 n+2}{n-1}}\left(\mathbb{R}^{n} \times I\right)}+\left\|U_{t} f_{j}^{3}\right\|_{L^{\frac{2 n+2}{n-1}}\left(\mathbb{R}^{n} \times I\right)}\right)
\end{aligned}
$$

where the supports of the Fourier transforms of $f_{j}^{1}, f_{j}^{2}$ and $f_{j}^{3}$ are contained in the set $\left\{\xi \in \mathbb{R}^{n}:|\xi| \sim 2^{j}\right\}$, and $\left\|f_{j}^{1}\right\|_{p},\left\|f_{j}^{2}\right\|_{p}$ and $\left\|f_{j}^{3}\right\|_{p}$ are bounded by $C\|f\|_{p}$. By the Strichartz estimates and re-scaling we can see that for $l=1,2,3$,

$$
\left\|U_{t} f_{j}^{l}\right\|_{L^{\frac{2 n+2}{n-1}}\left(\mathbb{R}^{n} \times I\right)} \leq C 2^{j / 2}\|f\|_{2} .
$$

Putting this into (1.11) yields (1.10).

Finally, we mention that an analogue of Theorem 1.4 holds for the maximal operators associated with smoothly varying hypersurfaces, which were considered in [SS] (Theorem 4.1). This can be shown in the same way.

\section{Proof of Proposition 1.2}

This section is devoted to the proof of the sharp local smoothing estimate (1.2). It will be deduced from (1.3) and the following proposition. We only need to set $r_{0}=5 / 3$ in Proposition 2.1. 
Proposition 2.1. Let $I$ be the interval [1,2]. Suppose that (1.3) holds for $r \geq r_{0}$, $r_{0}<2$. Then for every $f$ with supp $\widehat{f} \subset\left\{\xi \in \mathbb{R}^{2}:|\xi| \sim N\right\}$,

$$
\left\|U_{t} f\right\|_{L^{q}\left(\mathbb{R}^{2} \times I\right)} \leq C N^{3 / 2-6 / q}\|f\|_{L^{p}\left(\mathbb{R}^{2}\right)}
$$

provided $\left(3+r_{0}\right)<q \leq \infty$ and $3 / q+1 / p=1$.

Let $f$ be a function with supp $\widehat{f} \subset\left\{\xi \in \mathbb{R}^{2}:|\xi| \sim N\right\}$. Without loss of generality we may assume the support of $\hat{f}$ is contained in a small conic neighborhood of the direction $(1,0)$.

To exploit the bilinear estimates, we use a decomposition technique which was used in [TV2, section 4]. For each $j \geq 1$, let us dyadically divide the circle $S^{1}$ into $2^{j}$ arcs $I_{k}^{j}$ of length $\pi 2^{1-j}$. We will write $I_{k}^{j} \sim I_{k^{\prime}}^{j}$ to mean that $I_{k}^{j}$ and $I_{k^{\prime}}^{j}$ are not adjacent but have adjacent parent arcs of length $\pi 2^{2-j}$. Then, by a Whitney decomposition of $S^{1} \times S^{1}$ away from the diagonal $D$ of $S^{1} \times S^{1}$ (see [TVV]), we have

$$
S^{1} \times S^{1} \backslash D=\bigcup_{j \geq 1} \bigcup_{\left(k, k^{\prime}\right): I_{k}^{j} \sim I_{k^{\prime}}^{j}} I_{k}^{j} \times I_{k^{\prime}}^{j}
$$

Let $\psi$ be a smooth function in $C_{0}^{\infty}([1 / 2,4])$ satisfying $\psi(x)=1$ if $1 \leq x \leq 2$. Let $f_{k}^{j}$ be given in polar coordinates by

$$
\widehat{f_{k}^{j}}(r, \theta)=\widehat{f}(r, \theta) \chi_{I_{k}^{j}}(\theta) \psi(r / N) .
$$

Since the support of $\widehat{f}$ is contained in a small conic neighborhood of the direction $(1,0)$, we may assume that all the $I_{k}^{j}$ are contained in a small neighborhood of $(1,0)$. Trivially, it follows that

$$
U_{t} f(x) \cdot U_{t} f(x)=\sum_{j \geq 1} \sum_{\left(k, k^{\prime}\right): I_{k}^{j} \sim I_{k^{\prime}}^{j}} U_{t} f_{k}^{j}(x) \cdot U_{t} f_{k^{\prime}}^{j}(x) .
$$

Thus, it is more convenient to consider a bilinear operator than a linear one. For each $j \geq 1$, define a bilinear operator $B_{j}^{N}$ by

$$
B_{j}^{N}(f, g)(x)=\sum_{I_{k}^{j} \sim I_{k^{\prime}}^{j}} U_{t} f_{k}^{j}(x) \cdot U_{t} g_{k^{\prime}}^{j}(x) .
$$

We want to compute the operator norm of $B_{j}^{N}$ from $L^{p}\left(\mathbb{R}^{2}\right) \times L^{p}\left(\mathbb{R}^{2}\right)$ to $L^{q / 2}\left(\mathbb{R}^{2} \times I\right)$.

Lemma 2.2. Suppose for some $p, q$ satisfying $4 \leq q, 2 \leq p$ and $1 / 2 \leq 1 / q+1 / p$, there is a constant $B$ such that if $I_{k}^{j} \sim I_{k^{\prime}}^{j}$, then

$$
\left\|U_{t} f_{k}^{j} \cdot U_{t} g_{k^{\prime}}^{j}\right\|_{L^{\frac{q}{2}}\left(\mathbb{R}^{2} \times I\right)} \leq B\left\|f_{k}^{j}\right\|_{L^{p}\left(\mathbb{R}^{2}\right)}\left\|g_{k^{\prime}}^{j}\right\|_{L^{p}\left(\mathbb{R}^{2}\right)} .
$$

Then there is a constant $C$, independent of $j$ and $N$, such that

$$
\left\|B_{j}^{N}(f, g)\right\|_{L^{\frac{q}{2}}\left(\mathbb{R}^{2} \times I\right)} \leq C B\|f\|_{L^{p}\left(\mathbb{R}^{2}\right)}\|g\|_{L^{p}\left(\mathbb{R}^{2}\right)} .
$$

Proof. Since the $I_{k}^{j}$ are contained in a small neighborhood of $(1,0)$, it is easy to see that if $I_{k}^{j} \sim I_{k^{\prime}}^{j}$, then supp $\widehat{f}_{k}^{j}+\operatorname{supp} \widehat{g}_{k}^{j}$ is contained in the set $\left\{\xi \in \mathbb{R}^{2}\right.$ : $\left.\operatorname{dist}\left(\xi /|\xi|, I_{k}^{j}\right) \leq 2^{4-j}\right\}$. From this we can see that the Fourier supports of $\left\{U_{t} f_{k}^{j}\right.$. $\left.U_{t} g_{k^{\prime}}^{j}\right\}_{\left(k, k^{\prime}\right): I_{k}^{j} \sim I_{k^{\prime}}^{j}}$ are contained in essentially disjoint rectangles and the overlap among these rectangles is uniformly bounded in $j$. Now we use a lemma in TV2, which can be proven using Plancherel's theorem and a standard argument. 
Lemma 2.3 ([TV2, Lemma 7.1). Let $\left\{R_{k}\right\}$ be a collection of rectangles in frequency space such that the dilates $\left\{2 R_{k}\right\}$ are essentially disjoint, and suppose that $\left\{F_{k}\right\}$ are a collection of functions whose Fourier supports are contained in $R_{k}$. Then for $1 \leq p \leq \infty$ we have

$$
\left(\sum_{k}\left\|F_{k}\right\|_{p}^{p^{*}}\right)^{1 / p^{*}} \lesssim\left\|\sum_{k} F_{k}\right\|_{p} \lesssim\left(\sum_{k}\left\|F_{k}\right\|_{p}^{p_{*}}\right)^{1 / p_{*}}
$$

where $p_{*}=\min \left(p, p^{\prime}\right)$ and $p^{*}=\max \left(p, p^{\prime}\right)$.

Using this and the observation made above, we have for $q / 2 \geq 2$,

$$
\left\|\sum_{I_{k}^{j} \sim I_{k^{\prime}}^{j}} U_{t} f_{k}^{j} \cdot U_{t} g_{k^{\prime}}^{j}\right\|_{L^{\frac{q}{2}\left(\mathbb{R}^{2}\right)}} \leq C\left(\sum_{I_{k}^{j} \sim I_{k^{\prime}}^{j}}\left\|U_{t} f_{k}^{j} \cdot U_{t} g_{k^{\prime}}^{j}\right\|_{L^{\frac{q}{2}}\left(\mathbb{R}^{2}\right)}^{\left(\frac{q}{q}\right)^{\prime}}\right)^{1-\frac{2}{q}}
$$

with $C$ independent of $j$ and $t$. After raising both sides to the power $q / 2$, we integrate on the interval $I$ to get

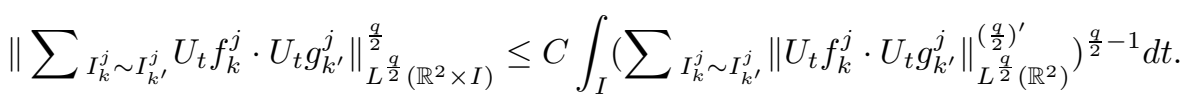

Since $q / 2 \geq 2$, by Minkowski's inequality

$$
\left\|B_{j}^{N}(f, g)\right\|_{L^{\frac{q}{2}}\left(\mathbb{R}^{2} \times I\right)} \leq C\left(\sum_{I_{k}^{j} \sim I_{k^{\prime}}^{j}}\left\|U_{t} f_{k}^{j} \cdot U_{t} g_{k^{\prime}}^{j}\right\|_{L^{\frac{q}{2}}\left(\mathbb{R}^{2} \times I\right)}^{\left(\frac{q}{q^{\prime}}\right)}\right)^{1-\frac{2}{q}} .
$$

From (2.2) we have $\left\|B_{j}^{N}(f, g)\right\|_{L^{\frac{q}{2}}\left(\mathbb{R}^{2} \times I\right)} \leq C B\left(\sum_{I_{k}^{j} \sim I_{k^{\prime}}^{j}}\left\|f_{k}^{j}\right\|_{L^{p}}^{\left(\frac{q}{2}\right)^{\prime}}\left\|g_{k^{\prime}}^{j}\right\|_{L^{p}}^{\left(\frac{q}{2}\right)^{\prime}}\right)^{1-\frac{2}{q}}$. Since there are at most four values of $k$ associated to each $k^{\prime}$, it follows from Schwarz's inequality and the condition $1 / 2-1 / q \leq 1 / p$ that

$$
\left\|B_{j}^{N}(f, g)\right\|_{L^{\frac{q}{2}}\left(\mathbb{R}^{2} \times I\right)} \leq C B\left(\sum_{k}\left\|f_{k}^{j}\right\|_{L^{p}}^{p}\right)^{1 / p}\left(\sum_{k}\left\|g_{k}^{j}\right\|_{L^{p}}^{p}\right)^{1 / p} .
$$

Now it is sufficient to show $\left(\sum_{k}\left\|f_{k}^{j}\right\|_{L^{p}}^{p}\right)^{1 / p} \leq C\|f\|_{L^{p}}$ for $p \geq 2$. But this follows from Lemma 2.3, because the Fourier supports of $f_{k}^{j}$ are contained in essentially disjoint rectangles.

In view of Lemma 2.2, to compute the norm of $B_{j}^{N}$ it is sufficient to consider $U_{t} f_{k}^{j} \cdot U_{t} g_{k^{\prime}}^{j}$ when $I_{k}^{j} \sim I_{k^{\prime}}^{j}$. This will be done by treating the cases $2^{j}>N^{\frac{1}{2}}$ and $2^{j} \leq N^{\frac{1}{2}}$ separately. By rotation, we may assume that $\widehat{f}_{k}^{j}$ and $\widehat{g}_{k^{\prime}}^{j}$ are supported on the sets

$$
\left\{\xi \in \mathbb{R}^{2}: \xi_{1} \sim N,\left|\xi_{2}\right| \sim N 2^{-j}\right\}, \quad\left\{\xi \in \mathbb{R}^{2}: \eta_{1} \sim N,\left|\xi_{2}\right| \ll N 2^{-j}\right\},
$$

respectively.

First we claim that if $2^{j}>N^{\frac{1}{2}}$, then for $p \leq q, 1 / p+3 / q=1$,

$$
\left\|U_{t} f_{k}^{j} \cdot U_{t} g_{k^{\prime}}^{j}\right\|_{L^{q / 2}\left(\mathbb{R}^{2} \times I\right)} \leq C N^{(3-12 / q)} 2^{-j(1-4 / q)}\left\|f_{k}^{j}\right\|_{p}\left\|g_{k^{\prime}}^{j}\right\|_{p} .
$$

To see this, note that $\widehat{f}_{k}^{j}$ is supported in a rectangle of size $N \times N 2^{-j}$. By dilation $\left(\xi_{1}, \xi_{2}\right) \rightarrow\left(N \xi_{1}, N 2^{-j} \xi_{2}\right)$

$$
U_{t} f_{k}^{j}(x)=N^{2} 2^{j} \int_{\mathbb{R}^{2}} e^{i\left\langle x,\left(N \xi_{1}, N 2^{-j} \xi_{2}\right)\right\rangle+i t\left|\left(N \xi_{1}, N 2^{-j} \xi_{2}\right)\right|} \beta\left(\xi_{1}, \xi_{2}\right) \widehat{f}_{k}^{j}\left(N \xi_{1}, N 2^{-j} \xi_{2}\right) d \xi
$$

where $\beta \in C_{0}^{\infty}([1 / 2,4] \times[-2,2])$ and $\beta \equiv 1$ on the set $[1,2] \times[-1,1]$. For $\xi \in$ $[1 / 2,4] \times[-2,2]$, we have

$$
t\left|\left(N \xi_{1}, N 2^{-j} \xi_{2}\right)\right|=t N \xi_{1} \sqrt{1+\left(2^{-j} \frac{\xi_{2}}{\xi_{1}}\right)^{2}}=t N \xi_{1}+O\left(t 2^{-2 j} N\right) .
$$


Since $2^{j}>N^{\frac{1}{2}}, e^{i O\left(t 2^{-2 j} N\right)} \beta$ is uniformly contained in $C_{0}^{\infty}([1 / 2,4] \times[-2,2])$, so it can be expanded in a Fourier series so that $e^{O\left(t 2^{-2 j} N\right)} \beta=\sum_{l \in \mathbb{Z}^{2}} C_{l}(t) e^{i\langle\xi, l\rangle}$ with $\sum_{l \in \mathbb{Z}^{2}}\left|C_{l}(t)\right|<M$ uniformly in $t$. By re-scaling, $\left(\xi_{1}, \xi_{2}\right) \rightarrow\left(\xi_{1} / N, 2^{j} \xi_{2} / N\right)$

$$
U_{t} f_{k}^{j}(x)=\sum_{l \in \mathbb{Z}^{2}} C_{l}(t) \int_{\mathbb{R}^{2}} e^{i\langle x, \xi\rangle+i t \xi_{1}} e^{i\left\langle\left(\xi_{1} / N, 2^{j} \xi_{2} / N\right), l\right\rangle} \widehat{f}_{k}^{j}(\xi) d \xi .
$$

This is essentially a sum of Fourier transforms. We need the following elementary lemma known as Bernstein's inequality.

Lemma 2.4. If $\widehat{f}$ is supported on a rectangle $Q$, then for $1 \leq p \leq q \leq \infty$

$$
\|f\|_{q} \leq|Q|^{\frac{1}{p}-\frac{1}{q}}\|f\|_{p} .
$$

Using this, we can easily see that for $1 \leq p \leq q$,

$$
\left\|U_{t} f_{k}^{j}\right\|_{L^{q}\left(\mathbb{R}^{2} \times I\right)} \leq C N^{2(1 / p-1 / q)} 2^{-j(1 / p-1 / q)}\left\|f_{k}^{j}\right\|_{p}
$$

because $\widehat{f}_{k}^{j}$ is supported in a rectangle of size $N \times N 2^{-j}$. In particular, if one sets $1 / p=1-3 / q$, then

$$
\left\|U_{t} f_{k}^{j}\right\|_{L^{q}\left(\mathbb{R}^{2} \times I\right)} \leq C N^{(3 / 2-6 / q)} 2^{-j(1 / 2-2 / q)}\left\|f_{k}^{j}\right\|_{p}
$$

because $2^{j} \geq N^{1 / 2}$ and $q \geq 4$. The same estimates also hold for $g_{k^{\prime}}^{j}$. Thus, by Hölder's equality, we can see that (2.4) follows. From Lemma 2.2 and (2.4), it follows that if $2^{j}>N^{1 / 2}$, then for $1 / p+3 / q=1, p \geq 2$ and $q \geq 4$,

$$
\left\|B_{j}^{N}(f, g)\right\|_{L^{q / 2}\left(\mathbb{R}^{2} \times I\right)} \leq C N^{(3-12 / q)} 2^{-(1-4 / q) j}\|f\|_{p}\|g\|_{p} .
$$

Now we turn to the case $2^{j} \leq N^{\frac{1}{2}}$. To begin with, we want to show that if $2^{j} \leq N^{\frac{1}{2}}$, then

$$
\left\|U_{t} f_{k}^{j} \cdot U_{t} g_{k^{\prime}}^{j}\right\|_{L^{\infty}\left(\mathbb{R}^{2} \times I\right)} \leq C N 2^{-2 j}\left\|f_{k}^{j}\right\|_{\infty}\left\|g_{k^{\prime}}^{j}\right\|_{\infty} .
$$

Dividing the $\operatorname{arc} I_{k}^{j}$ into $\sqrt{N} 2^{-j}$ sub-arcs $I_{k, n}^{j}$, we decompose $f_{k}^{j}$ into $\sqrt{N} 2^{-j}$ functions $\left\{f_{k, n}^{j}\right\}_{n}$ such that the $\widehat{f}_{k, n}^{j}$ are supported on rectangles of size $N \times N^{1 / 2}$, whose major directions are $\left(1, \theta_{n}\right)$. Then using the same method as in the previous case, for these decomposed functions we linearize the phase $t|\xi|$ to obtain

$$
U_{t} f_{k}^{j}(x)=\sum_{n} \sum_{l \in \mathbb{Z}^{2}} C_{l, n}(t) \int_{\mathbb{R}^{2}} e^{i\langle x, \xi\rangle} e^{i\left(t \xi_{1}+t \xi_{2} \theta_{n}\right)\left(1+\theta_{n}^{2}\right)^{-1 / 2}} e^{i\langle\xi / N, l\rangle} \widehat{f}_{k, n}^{j}(\xi) d \xi
$$

with $\sum_{l \in \mathbb{Z}^{2}}\left|C_{l, n}(t)\right|<M$ uniformly in $t, n$. Indeed, after the dilation $\xi \rightarrow N \xi$ on the support of $\widehat{f}_{k, n}^{j}(\cdot N)$, we have $t\left|\left(N \xi_{1}, N \xi_{2}\right)\right|=\left(t N \xi_{1}+t N \xi_{2} \theta_{n}\right) / \sqrt{1+\theta_{n}^{2}}+$ $N \xi_{1} O\left(\left(\xi_{2} / \xi_{1}-\theta_{n}\right)^{2}\right)$. Since the diameter of the angular support of $\widehat{f}_{k, n}^{j}(\cdot N)$ is about $N^{-1 / 2}$, we have $N \xi_{1} O\left(\left(\xi_{2} / \xi_{1}-\theta_{n}\right)^{2}\right)=O(1)$. Introducing a cut-off function $\beta$, we expand $\beta e^{i N \xi_{1} O\left(\left(\xi_{2} / \xi_{1}-\theta_{n}\right)^{2}\right)}$ in a Fourier series $\sum_{l \in \mathbb{Z}^{2}} C_{l, n}(t) e^{i\langle\xi, l\rangle}$ and re-scale by $\xi \rightarrow N \xi$ to get (2.7). Since the number of $n$ is about $\sqrt{N} 2^{-j}$, an application of Lemma 2.4 to each $f_{k, n}^{j}$ in $(2.7)$ gives

$$
\left\|U_{t} f_{k}^{j}\right\|_{L^{\infty}\left(\mathbb{R}^{2} \times I\right)} \leq C \sqrt{N} 2^{-j}\left\|f_{k}^{j}\right\|_{\infty}
$$

Trivially, the same estimate holds for $g_{k^{\prime}}^{j}$. Therefore, (2.6) follows.

Now we want to show that if $I_{k}^{j} \sim I_{k^{\prime}}^{j}$, then

$$
\left\|U_{t} f_{k}^{j} \cdot U_{t} g_{k^{\prime}}^{j}\right\|_{L^{r}\left(\mathbb{R}^{2} \times I\right)} \leq C N^{2-3 / r} 2^{j(3 / r-1)}\left\|f_{k}^{j}\right\|_{2}\left\|g_{k^{\prime}}^{j}\right\|_{2} .
$$


By re-scaling it suffices to show the following.

Lemma 2.5. Suppose (1.3) holds. If $\widehat{f}$ and $\widehat{g}$ are supported on the sets $\left\{\xi \in \mathbb{R}^{2}\right.$ : $\left.\xi_{1} \sim 1,\left|\xi_{2}\right| \sim 2^{-j}\right\}$ and $\left\{\xi \in \mathbb{R}^{2}: \xi_{1} \sim 1,\left|\xi_{2}\right| \ll 2^{-j}\right\}$, respectively, then

$$
\left\|U_{t} f \cdot U_{t} g\right\|_{L^{r}\left(\mathbb{R}^{2} \times \mathbb{R}\right)} \leq C 2^{j(3 / r-1)}\|f\|_{2}\|g\|_{2} .
$$

Proof. By Plancherel's theorem, it is sufficient to show

$$
\|\widehat{f d \mu} \widehat{g d \mu}\|_{r} \leq C 2^{-j} 2^{3 j / r}\|f\|_{2}\|g\|_{2}
$$

whenever $f$ and $g$ are supported on the sets $\left\{(\xi, \tau) \in \Gamma: \xi_{1} \sim 1,\left|\xi_{2}\right| \sim 2^{-j}\right\}$ and $\left\{(\xi, \tau) \in \Gamma: \xi_{1} \sim 1,\left|\xi_{2}\right| \ll 2^{-j}\right\}$, respectively. To obtain this from the bilinear cone restriction estimate, observe that the cone $\Gamma$ can be rotated without affecting the estimate (1.3). Now make a coordinate transform given by

$$
\eta_{1}=\frac{\tau+\xi_{1}}{\sqrt{2}}, \quad \eta_{2}=\frac{\tau-\xi_{1}}{\sqrt{2}}, \quad \rho=\xi_{2} .
$$

This rotation will move the forward light cone $\Gamma$ to the cone $\widetilde{\Gamma}$ which is tangent to the $\eta_{2}$-axis. Note that the defining equation for $\Gamma$ (i.e. $\tau^{2}=\xi_{1}^{2}+\xi_{2}^{2}, \tau \sim 1$ ) is transformed to $2 \eta_{1} \eta_{2}=\rho^{2}$ with $\eta_{1} \sim 1$. Therefore, $\widehat{f d \mu}$ is essentially the same as

$$
T f(x, t)=\int_{\widetilde{\Gamma}} e^{i\left\langle x,\left(\eta_{1}, \rho\right)\right\rangle+i t \rho^{2} / 2 \eta_{1}} f(\eta, \rho) d \widetilde{\mu}(\eta, \rho)
$$

where $d \widetilde{\mu}$ is the surface measure on $\widetilde{\Gamma}$. It is sufficient to consider $T f \cdot T g$ instead of $\widehat{f d \mu} \widehat{g d \mu}$. In $(\eta, \rho)$-coordinates the conditions imposed on the supports of $f, g$ should be read as: the supports of $f, g$ are contained in $\left\{(\eta, \rho) \in \widetilde{\Gamma}: \eta_{1} \sim 1, \rho \sim\right.$ $\left.2^{-j}\right\},\left\{(\eta, \rho) \in \widetilde{\Gamma}: \eta_{1} \sim 1,|\rho| \ll 2^{-j}\right\}$, respectively. Now make the change of variables $\rho \rightarrow 2^{-j} \rho$ to get $T f(x, t)=2^{-j} T\left(f\left(\cdot, 2^{-j} \cdot\right)\right)\left(x_{1}, 2^{-j} x_{2}, 2^{-2 j} t\right)$. For the bilinear operator

$T f(x, t) \cdot T g(x, t)=2^{-2 j} T f\left(\cdot, 2^{-j} \cdot\right)\left(x_{1}, 2^{-j} x_{2}, 2^{-2 j} t\right) \cdot T g\left(\cdot, 2^{-j} \cdot\right)\left(x_{1}, 2^{-j} x_{2}, 2^{-2 j} t\right)$

the functions $f\left(\cdot, 2^{-j} \cdot\right)$ and $g\left(\cdot, 2^{-j} \cdot\right)$ have disjoint angular supports with distance comparable to 1 , contained in the cone $\widetilde{\Gamma}$. Therefore, by the bilinear cone restriction estimate (1.3) we see that $\left\|T f\left(\cdot, 2^{-j} \cdot\right) \cdot T g\left(\cdot, 2^{-j} \cdot\right)\right\|_{r} \leq C 2^{j}\|f\|_{2}\|g\|_{2}$. Now, by rescaling it follows that

$$
\|T f \cdot T g\|_{r} \leq C 2^{-j} 2^{3 j / r}\|f\|_{2}\|g\|_{2} .
$$

This completes the proof.

Since for some fixed $I_{k}^{j}$ and $I_{k^{\prime}}^{j}$, the expression $U_{t} f_{k}^{j} \cdot U_{t} g_{k^{\prime}}^{j}$ can be considered as a bilinear operator, an interpolation between (2.6) and (2.8) shows that if $I_{k}^{j} \sim I_{k^{\prime}}^{j}$, then for $p, q$ satisfying $q \geq 2 r$ and $1 / p=r / q$,

$$
\left\|U_{t} f_{k}^{j} U_{t} g_{k^{\prime}}^{j}\right\|_{q / 2} \leq C N^{-6 / q+2 / p+1} 2^{j(6 / q+2 / p-2)}\left\|f_{k}^{j}\right\|_{p}\left\|g_{k^{\prime}}^{j}\right\|_{p}
$$

with $C$ independent of $I_{k}^{j}, I_{k^{\prime}}^{j}$. Therefore, from Lemma 2.2 and the above it follows that if $2^{j} \leq N^{1 / 2}$, then for $p, q$ satisfying $q \geq 2 r, 1 / p=r / q$, and $1 / p+1 / q \geq 1 / 2$, $p \geq 2, q \geq 4$,

$$
\left\|B_{j}^{N}(f, g)\right\|_{L^{q / 2}} \leq C N^{-6 / q+2 / p+1} 2^{j(6 / q+2 / p-2)}\|f\|_{p}\|g\|_{p} .
$$


To sum up the last estimates, we use the following lemma which is a multilinear extension of a result implicit in [B3]. An explicit statement can also be found in CSW]. We denote by $L^{p, r}$ the Lorentz spaces.

Lemma 2.6 (An interpolation lemma). Let $\varepsilon_{1}, \varepsilon_{2}>0$. Suppose that $\left\{T_{j}\right\}$ is a sequence of $n$-linear (or sublinear) operators such that for some $1 \leq p_{1}^{i}, p_{2}^{i}<\infty$, $i=1, \ldots n$ and $1 \leq q_{1}, q_{2}<\infty$,

$\left\|T_{j}\left(f^{1}, \ldots, f^{n}\right)\right\|_{q_{1}} \leq M_{1} 2^{\varepsilon_{1} j} \prod\left\|f^{i}\right\|_{p_{1}^{i}}, \quad\left\|T_{j}\left(f^{1}, \ldots, f^{n}\right)\right\|_{q_{2}} \leq M_{2} 2^{-\varepsilon_{2} j} \prod\left\|f^{i}\right\|_{p_{2}^{i}}$.

Then $T=\sum T_{j}$ is bounded from $L^{p^{1}, 1} \times \cdots \times L^{p^{n}, 1}$ to $L^{q, \infty}$ with

$$
\left\|T\left(f^{1}, \ldots, f^{n}\right)\right\|_{L^{q, \infty}} \leq C M_{1}^{\theta} M_{2}^{1-\theta} \prod\left\|f^{i}\right\|_{L^{p^{i}, 1}}
$$

where $\theta=\varepsilon_{2} /\left(\varepsilon_{1}+\varepsilon_{2}\right), 1 / q=\theta / q_{1}+(1-\theta) / q_{2}, 1 / p^{i}=\theta / p_{1}^{i}+(1-\theta) / p_{2}^{i}$.

Proof of Lemma 2.6. Let $N \in \mathbb{Z}$, which will be chosen later. Let $E_{1}, \ldots, E_{n}$ be measurable sets and let $\lambda>0$. Set $T_{N}=\sum_{-\infty}^{N} T_{j}$ and $T^{N}=\sum_{N+1}^{\infty} T_{j}$. Note

$$
\left\|T_{N}\right\|_{L^{p_{1}^{1}} \times \cdots \times L^{p_{1}^{n}} \rightarrow L^{q_{1}}} \leq C M_{1} 2^{N \varepsilon_{1}}, \quad\left\|T_{N}\right\|_{L^{p_{2}^{1}} \times \cdots \times L^{p_{2}^{n}} \rightarrow L^{q_{2}}} \leq C M_{2} 2^{-N \varepsilon_{2}}
$$

and $\left|\left\{x:\left|T\left(\chi_{E_{1}}, \ldots, \chi_{E_{n}}\right)(x)\right|>\lambda\right\}\right| \leq\left|\left\{x:\left|T_{N}\left(\chi_{E_{1}}, \ldots, \chi_{E_{n}}\right)(x)\right|>\frac{1}{2} \lambda\right\}\right|+\mid\{x:$ $\left.\left|T^{N}\left(\chi_{E_{1}}, \ldots, \chi_{E_{n}}\right)(x)\right|>\frac{1}{2} \lambda\right\} \mid$. By Tchebyshev's inequality, the measure of the set $\left\{x:\left|T\left(\chi_{E_{1}}, \ldots, \chi_{E_{n}}\right)(x)\right|>\lambda\right\}$ is bounded above by

$$
C\left(M_{1}^{q_{1}} 2^{\varepsilon_{1} N q_{1}} \prod\left|E_{i}\right|^{q_{1} / p_{1}^{i}} \lambda^{-q_{1}}+M_{2}^{q_{2}} 2^{-\varepsilon_{2} N q_{2}} \prod\left|E_{i}\right|^{q_{2} / p_{2}^{i}} \lambda^{-q_{2}}\right) .
$$

Now choosing $N$ which optimizes this yields

$$
\left|\left\{x:\left|T\left(\chi_{E_{1}}, \ldots, \chi_{E_{n}}\right)(x)\right|>\lambda\right\}\right| \leq C\left(M_{1}^{\theta} M_{2}^{1-\theta} \prod\left|E_{i}\right|^{1 / p^{i}} \lambda^{-1}\right)^{q} .
$$

This completes the proof.

Using Lemma 2.6 and (2.9), we see that if $1 / p+3 / q=1$ and $q=r+3$ (note that $4<q \leq 5$ and the conditions imposed on $p, q$ in (2.9) are satisfied),

$$
\left\|\sum 2_{2^{j} \leq N^{1 / 2}} B_{j}^{N}(f, g)\right\|_{L^{q / 2, \infty}\left(\mathbb{R}^{2} \times I\right)} \leq C N^{3-12 / q}\|f\|_{p, 1}\|g\|_{p, 1} .
$$

Indeed, observe that in (2.9) the exponent on $2^{j}$ is negative if $3 / q+1 / p>1$, and positive if $3 / q+1 / p<1$. Use Lemma 2.6 , and solve the conditions $1 / p+3 / q=1$ and $1 / p=r / q$ to get (2.10) (which is an estimate at the point of intersection of these two lines). Since (1.3) holds for $r_{0} \leq r$, it follows that for $1 / p+3 / q=1$ and $r_{0}+3 \leq q \leq 5$

$$
\left\|\sum_{2^{j} \leq N^{1 / 2}} B_{j}^{N}(f, g)\right\|_{L^{q / 2, \infty}\left(\mathbb{R}^{2} \times I\right)} \leq C N^{3-12 / q}\|f\|_{p, 1}\|g\|_{p, 1} .
$$

Since $q / 2>2$, by real interpolation for bilinear operators between these estimates (note that $r_{0}+3<5$ ), the $L^{q / 2, \infty}$-norm in the left-hand side can be replaced by an $L^{q / 2}$-norm (see [BL], Exercise 3.13.5). Therefore we have that for $1 / p+3 / q=1$ and $r_{0}+3<q<5$,

$$
\left\|\sum_{2^{j} \leq N^{1 / 2}} B_{j}^{N}(f, g)\right\|_{L^{q / 2}\left(\mathbb{R}^{2} \times I\right)} \leq C N^{3-12 / q}\|f\|_{p, 1}\|g\|_{p, 1} .
$$


Recall that $\left(U_{t} f\right)^{2}=\sum_{j \geq 1} B_{j}^{N}(f, f)$. Since $q>4$, by the last inequality and (2.5) we have

$$
\begin{aligned}
\left\|U_{t} f\right\|_{q}^{2} & \leq\left\|\sum_{2^{j}>N^{1 / 2}} B_{j}^{N}(f, f)\right\|_{q / 2}+\left\|\sum_{2^{j} \leq N^{1 / 2}} B_{j}^{N}(f, f)\right\|_{q / 2} \\
& \leq C N^{(3-12 / q)}\left(\sum_{2^{j} \geq N^{1 / 2}} 2^{-(1-4 / q) j}+1\right)\|f\|_{p, 1}^{2} \\
& \leq C N^{(3-12 / q)}\|f\|_{p, 1}^{2}
\end{aligned}
$$

provided that $1 / p+3 / q=1$ and $r_{0}+3<q<5$. This can be interpolated (via real interpolation) with the trivial estimate $\left\|U_{t} f\right\|_{L^{\infty}\left(\mathbb{R}^{\times} I\right)} \leq C N^{3 / 2}\|f\|_{1}$ to replace the $L^{p, 1}$-norm by an $L^{p}$-norm. This completes the proof of Proposition 2.1.

\section{ACKNOWLEDGEMENTS}

We thank the referee for many valuable comments and suggestions. These greatly improved the presentation of our results.

\section{REFERENCES}

[B1] J. Bourgain, Averages in the plane over convex curves and maximal operators, J. Analyse Math. 47 (1986), 69-85. MR 88f:42036

[B2] J. Bourgain, On high-dimensional maximal functions associated with convex bodies, Amer. J. Math. 108 (1986), 1467-1476. MR 88h:42020

[B3] J. Bourgain, Estimations de certaines functions maximales, C.R. Acad. Sci. Paris 301 (1985), 499-502. MR 87b:42023

[CSW] A. Carbery, A. Seeger, S. Wainger, and J. Wright, Class of singular integral operators along variable lines, Journal of Geometric Analysis 9 (1999), 583-605. MR 2001g:42026

[BL] J. Bergh and J. Löfström, Interpolation spaces: An introduction, Springer-Verlag, New York, 1976. MR 58:2349

[MSS] G. Mockenhaupt, A. Seeger and C.D. Sogge, Wave front sets, local smoothing and Bourgain's circular maximal theorem, Annals of Math. 136 (1992), 207-218. MR 93i:42009

[S] W. Schlag, A generalization of Bourgain's circular maximal theorem, Jour. Amer. Math. Soc. 10 (1997), 103-122. MR 97c:42035

[SS] W. Schlag and C. D. Sogge, Local smoothing estimates related to the circular maximal theorem, Math. Res. Let. 4 (1997), 1-15. MR 98e:42018

[SW] E.M. Stein and G. Weiss, Introduction to Fourier analysis on Euclidean spaces, Princeton University Press, 1971. MR 46:4102

[T] T. Tao, Endpoint bilinear restriction theorems for the cone and some sharp null form estimates, to appear, Math. Z.

[TVV] T. Tao, A. Vargas, L. Vega, A bilinear approach to the restriction and Kekeya conjectures, J. Amer. Math. Soc. 11 (1998), 967-1000. MR 99f:42026

[TV1] T. Tao, A.Vargas, A bilinear approach to cone multipliers. I, Geometric and functional analysis 10 (2000), 185-215. MR 2002e:42012

[TV2] T. Tao, A.Vargas, A bilinear approach to cone multipliers. II, Geometric and functional analysis 10 (2000), 216-258. MR 2002e:42013

[W] T. Wolff, A sharp cone restriction estimate, Annals of Math. 153 (2001), 661-698.

Department of Mathematics, Pohang University of Science and Technology, Pohang 790-784, KoreA

E-mail address: huk@euclid.postech.ac.kr 